\title{
INTELLEKTUAL TIZIMLAR TUSHUNCHASIGA NAZARIY YONDASHUV TAMOYILI
}

\author{
Kuldasheva Feruza Kurdoshevna, \\ Moxitddinova Surayyo Akmalovna, \\ Toshkent kimyo-texnologiya instituti \\ qoshidagi akademik litseyi, \\ "Informatika" fani o'qituvchilari
}

Annotatsiya. Intellektual tizimlar fani bugungi kunda eng rivojlanayotgan fanlardan biri hisoblanadi. Mazkur maqolada Intellektual tizimlar nima, jarayon sifatidagi nazariy qarashlar to' $\mathrm{g}$ 'risida fikr yuritadi. Zero, shiddat bilan rivojlanayotgan kunlarimizda, zamonaviy axborot kommunikatsiya texnologiyalari yordamida barcha ishlab chiqarish korxonalarida, transportni boshqarish tizimida, meditsinada va halq xo'jaligidagi barcha sohalarda avtomatlashtirilgan boshqaruv tizimlari sun'iy intellektlar yordamida yaratilmoqda.

Kalit so'zlar: internet, intellekt, sun'iy intellekt, algoritm, algoritm ta'lim maqsadi.

\section{ТЕОРЕТИЧЕСКИЙ ПОДХОД К ПОНЯТИЮ ИНТЕЛЛЕКТУАЛЬНЫХ СИСТЕМ}

Аннотация. Наука об интеллектуальных системах сегодня является одной из самых передовых наук. В этой статье обсуждается, что такое интеллектуальные системы и теоретические перспективы как процесса. Потому что в наши стремительно развивающиеся дни с помощью современных информационных и коммуникационных технологий с помощью искусственного интеллекта создаются автоматизированные системы управления во всех отраслях, системах управления транспортом, медицине и во всех секторах экономики.

Ключевые слова: Интернет, интеллект, искусственный интеллект, алгоритм, цели обучения алгоритму.

\section{THEORETICAL APPROACH TO THE CONCEPT OF INTELLIGENT SYSTEMS}

Annotation. The science of intelligent systems is one of the most advanced sciences today. This article discusses what intelligent systems are and theoretical perspectives as a process. Because in our rapidly developing days, with the help of modern information and communication technologies, with the help of artificial 
intelligence, automated control systems are created in all industries, transport management systems, medicine and in all sectors of the economy.

Key words: Internet, intelligence, artificial intelligence, algorithm, learning goals for the algorithm.

Hozirgi kunda rivojlangan davlatlarda aqlliy ko'chalar, xaydovchisiz o'zi yurar transport vositalari, ishlab chiqarishda robotlar yordamida tayyor mahsulotlar chiqarilayotgani misol bo'la oladi.

Intellektual tizimlar - xisoblash texnikasini takomillashtiruvchilarning diqqat e'tibori yetuk ekspert tizimlarini yaratishga qaratilgan. Bu birinchidan, EHMda yoki shaxsiy kompyuterda (ShK) yechiladigan masalalar sinfini, EHMning mavjud imkoniyatlarini va undan foydalanuvchilar ishtiyoqini kengaytirish, ikkinchidan ulardan foydalana bilish, ShK va dasturlash sohasiga mansub bo'lmagan shaxsiy kompyuterlardan foydalanuvchilar bilan aloqasini soddalashtirishdir. Yetuk ShKlar topshiriqlarni kiritish va ijodiy jarayonni amalga oshirish, haqiqiy (tabiiy) til so'rovlarini qabul qilish, axborotni belgi, tasvir, signal ko'rinishida qayta ishlash, ShKdagi bor bilimlarni yig'ish imkoniyatiga ega. ShKda joylashtirilgan yetuk interfeys kompyuterlarining bir qismi bilangina tanish bo'lgan insonlarni mashinada ishlashiga yordam beradi. Shuning uchun yetuk EHMlarning kelajagi porloqdir.

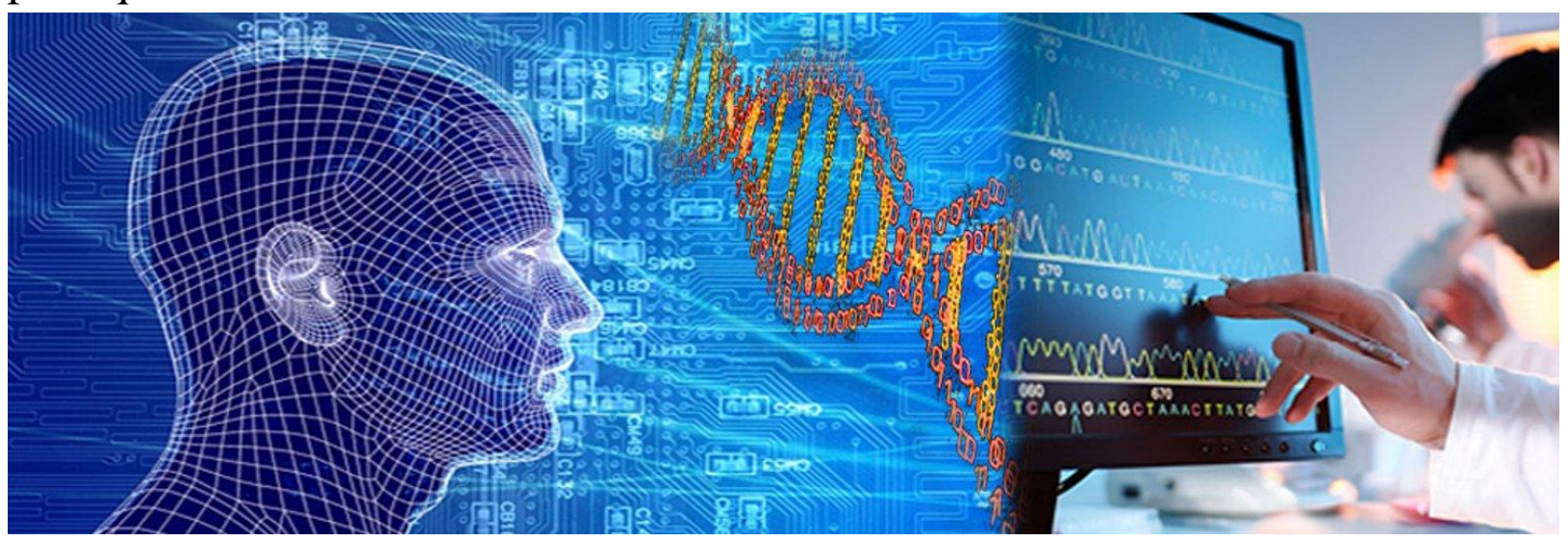

1-rasm.

«Itellektual tizimlar» fanini o'rganishi natijasida talaba yoki malakasini oshiruvchi shahs quyidagi bilim, malaka va ko'nikmaga ega bo'ladi: - bilimlar bazasining algoritmini yaratish; - zamonaviy kompyuterlarning imkoniyatlaridan foydalanib bazalar tuza olish; - yuqori darajadagi algoritmik tilda dasturlar tuza olish; - turli muhandislik masalalarini yecha olish; - tanlab olingan predmet soxa uchun intellektual tizim yarata bilish; - foydalanuchi uchun qulay interfeys tuza olish va boshqalar. Insonlarning zamonaviy kompyuterlarda turli masalalarni yechishi (algoritmlash va dasturlash), ulardan zamonaviy axborot texnologiyalar bo'lgan 
INTERNET, elektron pochta, kompyuter tarmoqlari, multemedia, grafik dasturlarida ishlashi mumkin.

Asosiy terminlar va tushunchalar. "Intellekt" (intelligence) termini lotinchadan (intellectus) so 'zidan kelib chiqib aql, idrok, fikr, mulohaza, ya'ni insonning fikrlash qobiliyati ma'nosini anglatadi. Intellekt deb inson miyasining intelektual masalalarni yechish qobiliyatiga aytiladi. Ushbu jarayon asosan tajriba asosida ma'lumotlarni qabul qilib, eslab qolish va maqsadli bilimlarni o'zgartirish, hamda har xil sharoitlarda moslashtirishga asoslangan bo'ladi. Yuqoridagi ta'rifda keltirilgan "bilim" so'zi insonning faqat sezish organlari orqali olinadigan ma'lumotlar emas, balki bizni qamrab olgan tabiyatdagi barcha ob'yektlar bir - biri bilan bog'liqligi hisoblanadi. Har bir inson turgan joyining atrofidagi barcha ko'zga ko'ringan va eshitayotgan ma'lumotlarni miyasida saqlaydi. Shuning uchun bilim - bu amaliyotda tekshirilgan va mantiqiy aniqlangan haqiqat fikr natijasi hisoblanadi. Asosan, bilim 7 insondagi tushuncha, fikrlash va nazariya hisoblanadi, hamda hayot jarayonida o'rganish natijasida rivojlanib boradi. Sun'iy intellekt (artificial intelligence) esa - bu avtomatik tizimlarning o'ziga inson intellektining alohida funktsiyalarini bajara olish qobiliyatiga aytiladi. Masalan, oldin olingan tajriba asosida tanlab va tashqi ta'sirlarni tahlil etib optimal savolga javob qabul qilish hisoblanadi. Yuqorida keltirilganlarni amalga oshirish uchun va intellektual masala oddiy masalalardan farqini bilish uchun albatta "algoritm" terminidan foydalaniladi.

Sun'iy intellektni yaratish to ' $\mathrm{g}$ 'risidagi nazariy izlanishlar XIII asrda yashagan ispan faylasufi, matematiki va shoir bo'lgan Raymond Lulliy har xil masalalarni yechadigan mexanik mashina yaratishga xarakat qilgan. XVIII asrda yashagan Leybnits va Dekart bir-biridan alohida universal til taklif etishgan. Lekin, asosan sun'iy intellekt ilmining yo'nalishi sifatida rasmiy kelib chiqishi XX asrning 40 yillariga to 'g'ri keladi, ya'ni EHM yaratila boshlagandan so'ng. Shu paytda Norbert Viner kibernetika faniga asos soladi. 1969 yilda Vashingtonning Dartsmut kolledjida sun'iy intellekt bo 'yicha 1- Xalqaro birlashgan konferentsiya bo 'lib o'tadi va "sun'iy intellekt" termini qonunlashtiriladi. Sun'iy intellektning asosiy yo"nalishlari: - Neyrokibernetika; - "Qora yashik (quti)" kibernetikasi. Neyrokibernetikaning asosi inson miyasi, ya'ni miyaga o'xshab fikrlash qobiliyatiga ega bo'ladi, chunki inson miyasi neyronlardan tashkil topgan. 12 Demak, fikrlay oladigan qurilma neyrokibernetika asosi hisoblanib, dasturiy va uskunaviy vositalardan iborat bo'ladi. Neyronlarni birlashmasi esa neyron tarmoqlari deb ataladi. Bunday neyron tarmoqlarni 1956 - 1965 yillarda Frenk 
Rozenblat va Mak-Kallakom yaratishadi, ya'ni inson ko'zini miya orqali bog'lanish modelini ishlab chiqishadi (alfavitdagi harflarni ko'rib o'qiy oladigan modeli edi). Kibernetikaning "qora yashik" uchun esa qurilmaning fikrlash uskunasi qanday bo 'lishidan tashqari, har hil savollarga odam miyasiga o"xshab javob bera oladigan edi. 1956 - 1963 yillarda insonning fikrlashiga o'xshagan modellar, algoritmlar va dasturiy vositalar yaratilishi faollashgan.

\section{Foydalanilgan adabiyotlar.}

1. Orifjonov M., Bekmuradov T., Xojimatova G., "Ekspert sistemalar", Amaliyot Toshkent. "Fan". 1971y.

2. Korneyev V.V., Garev A.F. i dr. Bazi dannix. Intellektualnaya obrabotka informatsii. - M.: «Nolidj», 2000.

3. Gavrilova T.A., Xoroshevskiy V.F. Bazi znaniy intellektualnix sistem. - S-Pb: Piter, 2001. 131

4. Zmitrovich A. I. Intellektualnie informatsionnie sistemi.- Mn: Tetra Sistems, 1977. 